\title{
Utilisation des membranes minérales Carbosep en industrie agro-alimentaire
}

\author{
par \\ R. VEYRE
}

\section{Ré s u m é}

Le procédé d'ultrafiltration Carbosep, caractérisé par l'utilisation des membranes minérales dites de $3^{\mathrm{e}}$ génération, a été développé sur de nouvelles lignes de produits en industrie agro-alimentaire. Les caractéristiques spécifiques des membranes et équipements Carbosep sont présentées.

Deux nouveaux procédés sont décrits :

- la clarification des jus de fruits appliquée aux jus de pommes;

- la microfiltration tangentielle des boissons alcoolisées fermentées appliquée aux vins.

Le rôle des paramètres directeurs des procédés sont examinés.

\section{S u $\mathbf{m} \mathbf{m}$ ar y \\ CARBOSEP ULTRAFILTRATION INORGANIC MEMBRANES \\ IN TE FOOD INDUSTRY}

The Carbosep Ultrafiltration process, which is using 3rd generation inorganic membranes, is already wellknow for applications in the Dairy Industry.

New lines of applications have been developped in the food industry. Technical data of Carbosep membranes and systems are presented.

Two new applications are described:

S.F.E.C., B.P. $\mathrm{n}^{\circ} 33$ - 84500 Bollène. 
- fruit juice clarification (as applied to apple juice);

- tangential microfiltration of fermented alcoholic beverages (as applied to wines).

Influence of processes main parameters are examined.

\section{INTRODUCTION}

Les membranes d'ultrafiltration de nature minérale commercialisées sous la marque Carbosep sont aujourd'hui internationalement connues, en particulier, dans les domaines des applications laitières et biotechnologiques. Les premières références industrielles européennes concrétisées en 1983 ont permis d'engager un développement commercial à l'échelle mondiale de ce procédé.

Indépendamment de l'essor industriel obtenu dans les domaines maintenant traditionnels de la standardisation et de la concentration protéique des laits ou de la concentration purification des protéines des lactosérums, S.F.E.C. a développé et industrialisé de nouveaux procédés qui font l'objet de la présente communication. Avant d'aborder la description de tels procédés, il est utile de rappeler les propriétés et les critères déterminants des membranes minérales et systèmes Carbosep.

\section{PROCEDE D'ULTRAFILTRATION CARBOSEP}

\subsection{Membranes}

Les membranes minérales Carbosep résultent du concept du milieu minéral microporeux ultrafiltrant c'est-à-dire d'un milieu microporeux de morphologie composite ; ce caractère composite est obtenu par la superposition de milieux microporeux homogènes.

La figure 1 symbolise un tel concept comparativement aux membranes organiques de morphologie homogène et asymétrique.

La figure 2 montre le cliché de microscopie électronique d'une membrane Carbosep.

Le tableau 1 présente les caractéristiques générales des membranes minérales Carbosep.

Les performances apportées par ces membranes permettent d'assurer la fiabilité d'exploitation tant attendue et maintenant largement démontrée; il est nécessaire de souligner la capacité de ces membranes à subir les contraintes les plus sévères vis-à-vis des 


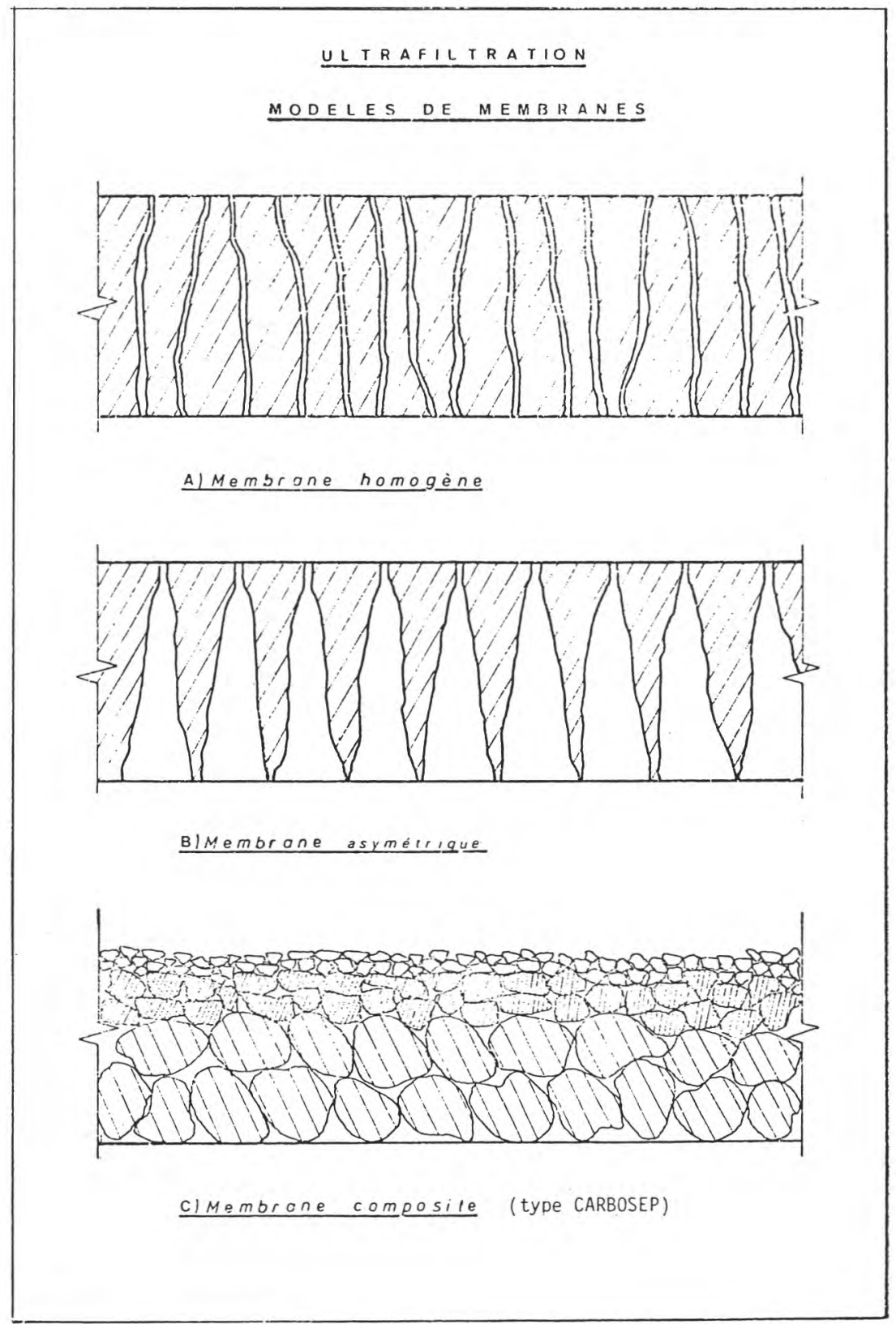

fig. 1 


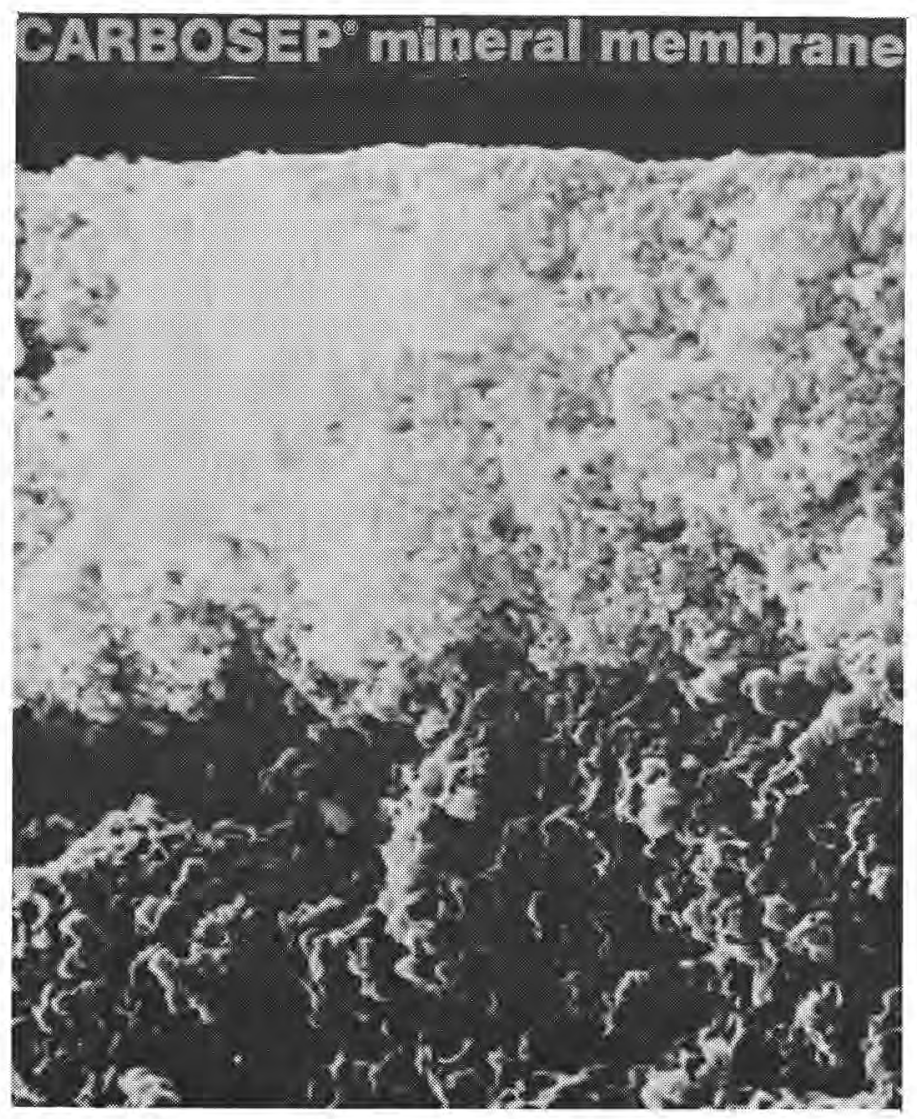

fig. 2

fluides procédés chauds et vis-à-vis des contraintes de stérilisation de type chimique et surtout de type vapeur.

\subsection{Modules}

Les membranes tubulaires sont assemblées en faisceaux parallèles dans des modules en acier inoxydable. La qualité des aciers est adaptée aux spécifications des industries agro-alimentaires et biotechnologiques. Le montage autorise l'interchangeabilité unitaire des membranes.

Les modules couvrent les surfaces diffusantes présentées dans le tableau 2 . 
TABLEAU 1

Caractéristiques générales des membranes minérales Carbosep

\begin{tabular}{|c|c|c|}
\hline Spécifications & Caractéristiques & Unités \\
\hline $\begin{array}{l}\text { - Support de membrane } \\
\text { - Membrane }\end{array}$ & $\begin{array}{l}\text { Carbone aggloméré } \\
\text { Oxyde métallique }\end{array}$ & \\
\hline $\begin{array}{l}\text { - Géométrie : } \\
\text { longueur } \\
\text { diamètre extérieur } \\
\text { diamètre intérieur }\end{array}$ & $\begin{array}{r}1200 \\
10 \\
6\end{array}$ & $\begin{array}{l}\mathrm{mm} \\
\mathrm{mm}\end{array}$ \\
\hline $\begin{array}{l}\text { - Mécanique : } \\
\text { pression d'éclatement } \\
\text { pression d'utilisation } \\
\text { force d'écrasement } \\
\text { module d'Young }\end{array}$ & $\begin{array}{r}60 \\
15 \\
30 \\
1000\end{array}$ & $\begin{array}{c}\text { bars } \\
\text { bars } \\
\mathrm{KgF} / 20 \mathrm{~mm}\end{array}$ \\
\hline $\begin{array}{l}\text { - Chimie : } \\
\text { pH d'utilisation (1) } \\
\text { stérilisation vapeur (2) } \\
\text { stérilisation oxydants }\end{array}$ & $\begin{array}{l}\text { \# } 0 \text { à } 14 \\
\text { oui } \\
\text { oui }\end{array}$ & \\
\hline $\begin{array}{l}\text { - Température d'utilisation procédé } \\
\text { jusqu'à }\end{array}$ & 150 & ${ }^{\circ} \mathrm{C}$ \\
\hline — Perméabilité à l'eau (3) & 180 à 600 & $1 / \mathrm{hm}^{2}$ \\
\hline $\begin{array}{l}\text { - Pouvoir de coupure : } \\
\text { référence M4 } \\
\text { référence M1 }\end{array}$ & $\begin{array}{l}20000 \\
50000\end{array}$ & $\begin{array}{l}\text { Dalton } \\
\text { Dalton }\end{array}$ \\
\hline $\begin{array}{l}\text { - Membrane de microfiltration (4) } \\
\text { référence M6 } \\
\text { diamètre pores }\end{array}$ & $\begin{array}{l}>2000000 \\
\quad \# 0,1\end{array}$ & $\begin{array}{c}\text { Dalton } \\
\mu\end{array}$ \\
\hline $\begin{array}{l}\text { - Toxicologie : } \\
\text { Agrément du Conseil Supérieur de } \\
\text { l'Hygiène de France } \\
\text { Agrément République Fédérale } \\
\text { d'Allemagne }\end{array}$ & $\begin{array}{l}\text { oui } \\
\text { oui }\end{array}$ & \\
\hline
\end{tabular}

(1) A toutes températures comprises entre 20 et $90^{\circ} \mathrm{C}$.

(2) Vapeur d'eau à $121^{\circ} \mathrm{C}$ sous 2 bars durant $30 \mathrm{~min}$.

(3) Mesurée à l'eau à $25^{\circ} \mathrm{C}$ sous pression moyenne 4 bars.

(4) Membranes de microfiltration caractérisées par la texture $\varnothing$ pores \# $0,1 \mu$. 
TABLEAU 2

Surface diffusante des modules Carbosep

\begin{tabular}{c|c}
\hline Référence module & Surface diffusante en $\mathrm{m}^{2}$ \\
\hline S 1 & 0,02 \\
S 7 & 0,16 \\
S 37 & 0,83 \\
S 151 & 3,40 \\
S 252 & 5,70 \\
\hline
\end{tabular}

\subsection{Systèmes}

Les systèmes industriels sont de type « multiétagés ». Ils fonctionnent en régime continu avec boucle de recirculation selon le schéma fonctionnel présenté figure 3.

Les équipements tendent progressivement vers une standardisation depuis l'équipement laboratoire jusqu'à l'équipement industriel de plusieurs centaines de $\mathrm{m}^{2}$ de surface diffusante.

La figure 4 montre les clichés de différents équipements Carbosep: type laboratoire, type pilote industriel, type unité industrielle monoétagée, type unité industrielle multiétagée.

Il est utile de préciser que S.F.E.C. dispose actuellement d'un parc pilote de 30 unités assurant les campagnes pilotes nécessaires à la définition des unités industrielles.

\section{CLARIFICATION DES JUS DE FRUITS}

Au cours de l'année 1983, les membranes minérales Carbosep ont connu un nouveau développement par la réalisation de 3 unités industrielles dans le domaine des jus de pommes.

La figure 5 montre la comparaison entre le procédé traditionnel et le procédé d'ultrafiltration.

Deux grands constats sont immédiatement perçus à l'examen des schémas de procédés. 


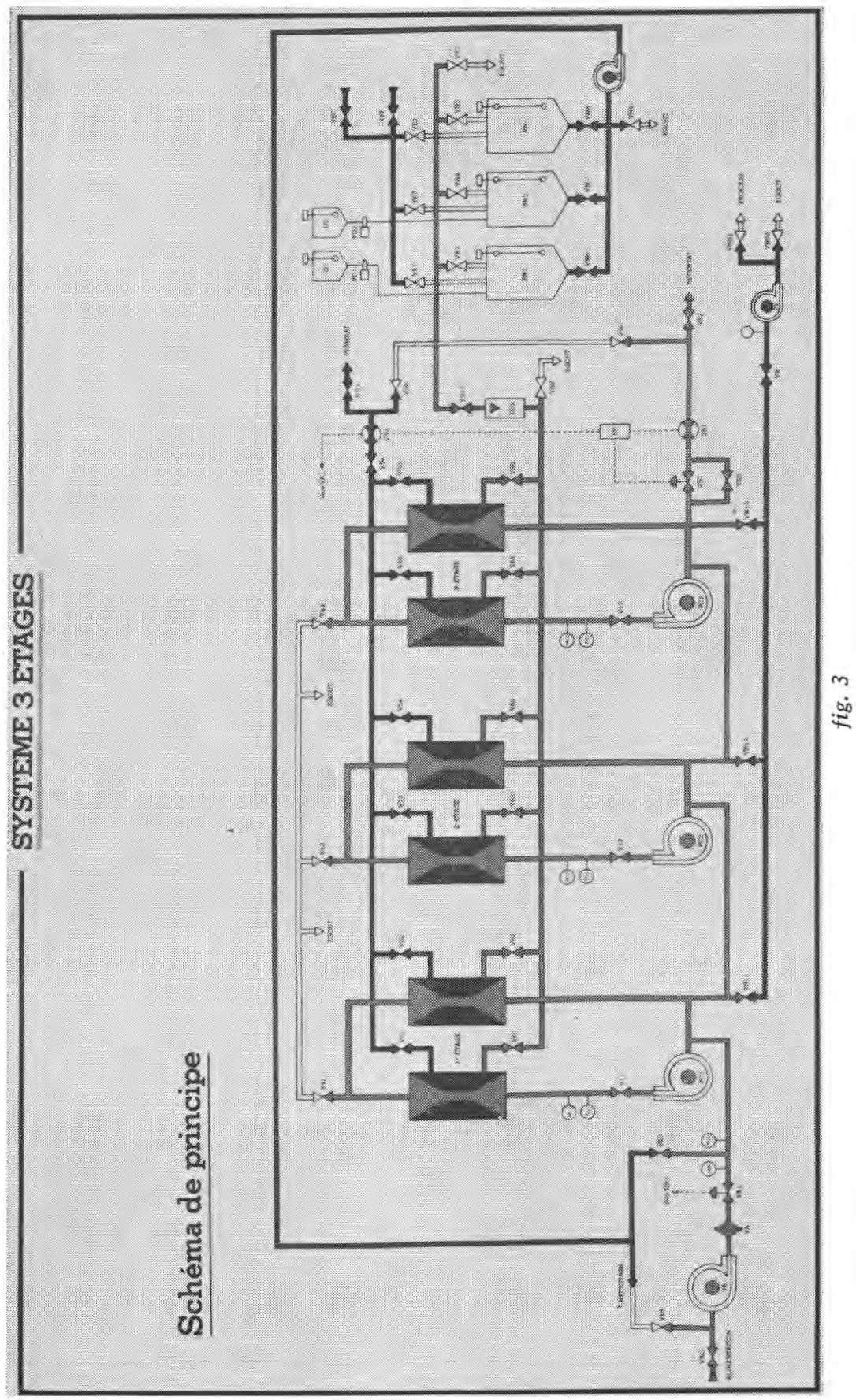




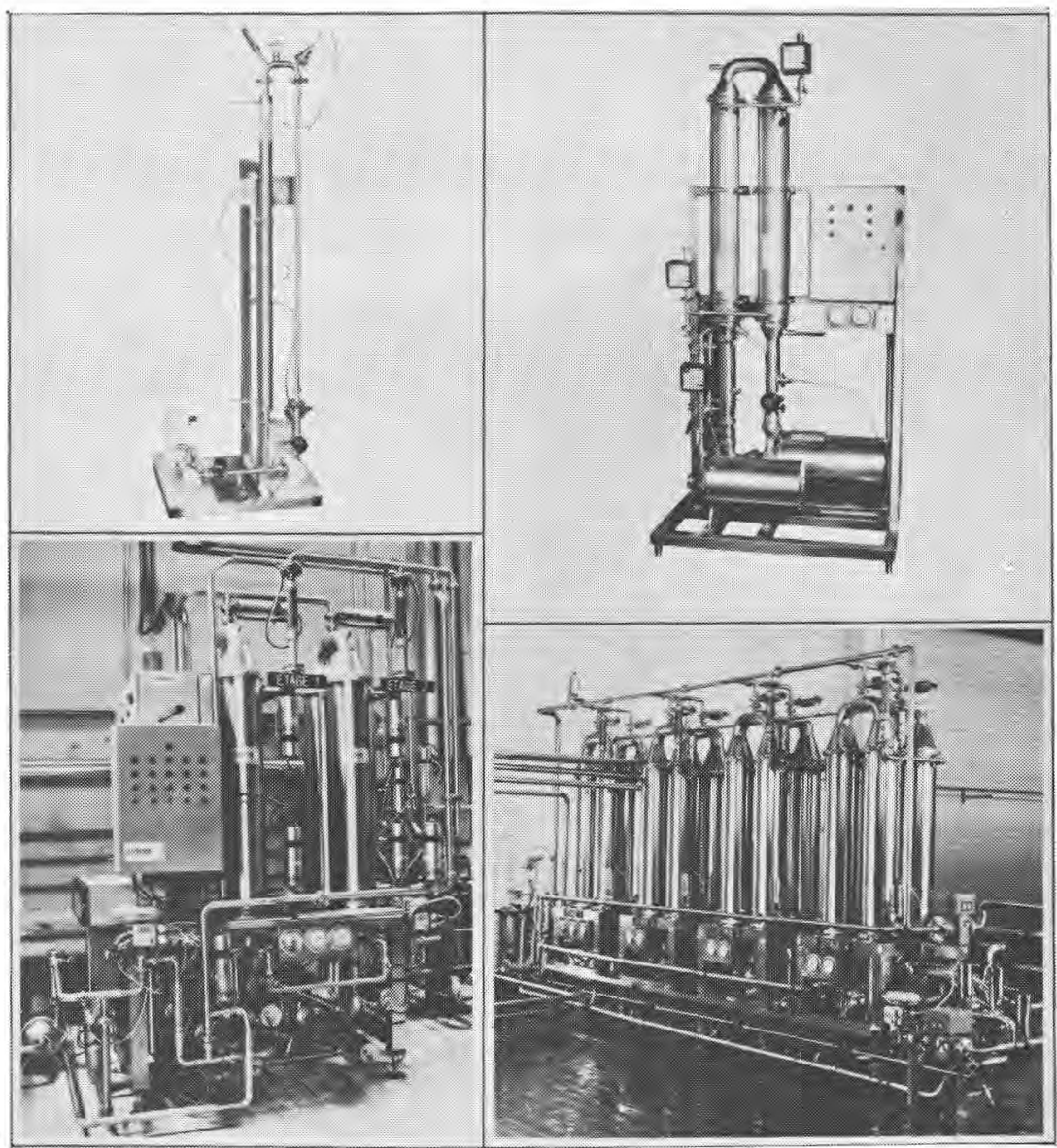

fig. 4

\section{Premier constat}

Simplification du procédé traditionnel de type discontinu par suppression de diverses opérations telles que : refroidissement, collage, débourbage des fonds de cuve, filtrations sur diatomées et plaques de cellulose.

\section{Deuxième constat}

Passage d'un procédé discontinu à un procédé continu facilitant les conditions d'exploitation. 


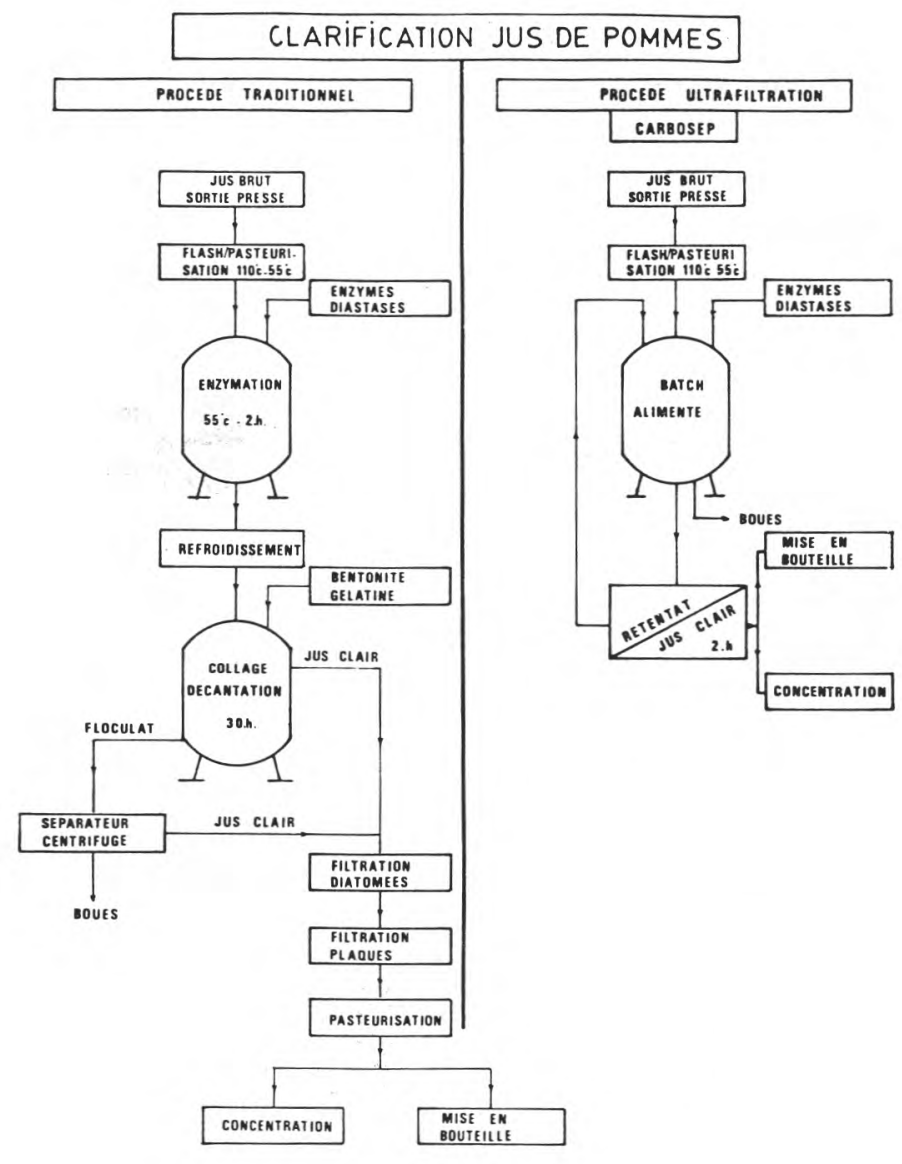

fig. 5

Ces deux constats ont pour conséquence un gain favorable au procédé d'ultrafiltration justifiant par là-même l'investissement de l'équipement. Le retour d'investissement est voisin de 18 mois à 2 ans selon les sites industriels.

Les économies sont réalisées au niveau :

- Matériel : diminution cuverie, suppression séparateur centrifuge et unités de filtration traditionnelle.

- Produits : diminution enzymation, suppression gélatine-bentonite, suppression kieselghur et plaques de cellulose. 
- Main d'œuvre : limitation des opérations de transfert, possibilité de mise en bouteille $2 \mathrm{~h}$ après la mise en route de l'unité d'ultrafiltration.

- Rendement matière : il a été évalué au minimum à $97 \%$ contre environ $90 \%$ à $93 \%$ en traditionnel selon les sites.

Les performances du procédé d'ultrafiltration, proprement dit, sont dépendantes d'un grand nombre de paramètres. Nous pouvons citer :

- Le choix du mode de fonctionnement : le régime type batch alimenté se révèle toujours le plus performant.

- Le taux, la température et la durée d'enzymation à l'aide de diastases spécifiques, variables selon l'origine et la maturité des pommes (cidre ou pommes à bouche).

- Le facteur de concentration dans la boucle d'ultrafiltration en régime établi associé d'une part aux taux de recyclage sur le batch et d'autre part à la capacité horaire de production.

- La température, la pression moyenne, la cinétique de mise en pression et la vitesse de circulation dans l'unité d'ultrafiltration conduisant à une perméabilité maximale.

- Le pouvoir de coupure des membranes déterminant la rétention enzymatique et l'indice de coloration du jus de pomme produit.

- Le facteur de concentration limite pour l'obtention d'un épuisement maximum du batch lié au rendement du procédé. Le facteur de concentration final est systématiquement $\geqslant 35$.

La détermination et le choix des paramètres précités ont permis d'obtenir une perméabilité moyenne par cycle de $22 \mathrm{~h}$ de $125 \mathrm{l} / \mathrm{hm}^{2}$ quelle que soit l'origine et la nature des pommes.

Quant à la qualité des jus de pommes produits par le procédé d'ultrafiltration elle est tout à fait comparable aux jus traditionnels.

A titre d'exemple le tableau 3 compare leur composition respective.

Nous pouvons noter malgré des compositions très voisines, deux particularités :

- Les jus ultrafiltrés conservent bien les tanins de la pomme alors que ceux-ci sont en partie complexés par le collage à la gélatine lors des traitements traditionnels.

- Les jus ultrafiltrés sont plus colorés que les jus traditionnels, l'opération de collage étant responsable de leur perte de couleur. Il faut également préciser que la couleur des jus ultrafiltrés est stable dans le temps contrairement aux jus traditionnels qui ont tendance au brunissement. Les tests de dégustation se sont révélés plus favorables aux jus clarifiés par ultrafiltration. 


\section{TABLEAU 3}

Comparaison des caractéristiques des jus de pommes clarifiés par le procédé traditionnel et le procédé d'ultrafiltration Carbosep

\begin{tabular}{|c|c|c|}
\hline Caractéristiques & $\begin{array}{l}\text { Clarification } \\
\text { traditionnelle }\end{array}$ & $\begin{array}{l}\text { Clarification par } \\
\text { ultrafiltration Carbosep }\end{array}$ \\
\hline $\begin{array}{lc}\begin{array}{l}\text { Densité (1) } \\
\text { Sucres totaux (2) }\end{array} & (\mathrm{g} / 1) \\
\text { Fructose } & (\mathrm{g} / 1) \\
\text { Glucose } & (\mathrm{g} / 1) \\
\text { Saccharose } & (\mathrm{g} / 1) \\
\text { Tanins (3) } & \mathrm{g} / 1) \\
\text { Matières sèches }(4) & (\%) \\
\text { Acidité totale (5) } & \\
\left.\text { (g H } \mathrm{SO}_{4} / 1\right) & \\
\text { Couleur }(6) & \\
\text { Turbidité (7) } & \end{array}$ & $\begin{array}{l}1,04437 \\
98,7 \\
67,8 \\
15,6 \\
15,3 \\
1,21 \\
11 \\
2,80 \\
\\
0,492 \\
0,057\end{array}$ & $\begin{array}{c}1,04488 \\
94,3 \\
60,0 \\
18,7 \\
15,6 \\
1,99 \\
11,2 \\
2,05\end{array}$ \\
\hline
\end{tabular}

(1) Densité : mesure ffeecutée à $20^{\circ} \mathrm{C}$ sur densimètre électronique De Paar.

(2) Sucres : méthode enzymatique Kit Boehringer.

(3) Tanins : méthode de Folin.

(4) Matières sèches : réfractométrie à $20^{\circ} \mathrm{C}$.

(5) Acidité : neutralisation à la soude jusqu'à $\mathrm{pH} 7$ exprimée en $\mathrm{g} / 1 \mathrm{H}_{2} \mathrm{SO}_{4}$.

(6) Couleur : mesurée au spectrophotomètre à $420 \mathrm{~nm}$.

(7) Turbidité : mesurée au spectrophotomètre à $650 \mathrm{~nm}$.

\section{MICROFILTRATION TANGENTIELLE DES BOISSONS ALCOOLISEES FERMENTEES PAR LE PROCEDE CARBOSEP}

Depuis 1983, S.F.E.C. a entrepris un programme de recherche et développement visant à valoriser le procédé Carbosep dans le domaine de la clarification des boissons alcoolisées fermentées dont les trois principales sont en France :

$$
\begin{aligned}
& \text { Le vin : } 50 \text { à } 80.10^{6} \mathrm{hl} . \\
& \text { La bière : } 20 \text { à } 25.10^{6} \mathrm{hl} \text {. } \\
& \text { Le cidre : } 15 \text { à } 20.10^{6} \mathrm{hl} \text {. }
\end{aligned}
$$

L'intérêt du procédé Carbosep sur une telle ligne de produits est identique à celui de son insertion sur la ligne de produits jus de fruits à savoir : passage d'un procédé discontinu à un procédé continu et simplification d'exploitation de la chaîne de filtration traditionnelle. Au cours de cette communication nous n'aborderons 
que le cas du vin sur lequel nous avons effectué un effort particulier en collaboration avec deux sections régionales de l'Institut Technique de la Vigne et du Vin.

I.T.V. : Section Régionale : Sud-Ouest, Rabastens.

I.T.V. : Centre Technique Expérimental : Villefranche-surSaône.

Différents travaux conduits sur la vinification des moûts microfiltrés feront l'objet d'une autre communication.

\section{MICROFILTRATION TANGENTIELLE DES VINS PAR LE PROCEDE CARBOSEP}

Il faut noter que nous avons adopté le terme de microfiltration tangentielle. En effet les diverses contraintes relatives aux vins tant au plan qualitatif qu'au plan économique, nous ont orientés sur le concept d'une membrane de microfiltration Carbosep généralisable aux boissons alcoolisées fermentées telles que le cidre et la bière. Il s'agit de la membrane maintenant commercialisée sous la référence M 6, de texture \# 0,1 $\mu$ présentée dans le tableau 1.

L'étude pratiquée sur des Beaujolais primeurs a pris en compte l'ensemble des paramètres de procédé dans de larges fourchettes de variations tels que : type de fonctionnement, choix de la texture membrane, rôle des paramètres directeurs, vitesse de circulation dans les boucles, pression, température, durée.

Le nouveau procédé de microfiltration tangentielle appliqué au Beaujolais primeur présente une perméabilité moyenne de 115 à $125 \mathrm{l} / \mathrm{hm}^{2}$ durant plusieurs heures de fonctionnement continu.

Un fonctionnement longue durée voisin de $90 \mathrm{~h}$ a été pratiqué sans nettoyage intermédiaire.

En ce qui concerne la qualité des vins obtenus par application du procédé optimisé, elle est très sensiblement identique à la qualité du vin traité par la méthode traditionnelle.

Les tableaux 4, 5 présentent les caractéristiques comparées entre :

- Le vin brut.

- Le vin filtré traditionnel.

- Le vin microfiltré par le procédé Carbosep.

Les résultats présentés dans les tableaux ci-après appellent les remarques suivantes :

- Les caractéristiques de turbidité et de filtrabilité sont considérablement améliorées par le procédé de microfiltration tangentielle Carbosep. 
TABLEAU 4

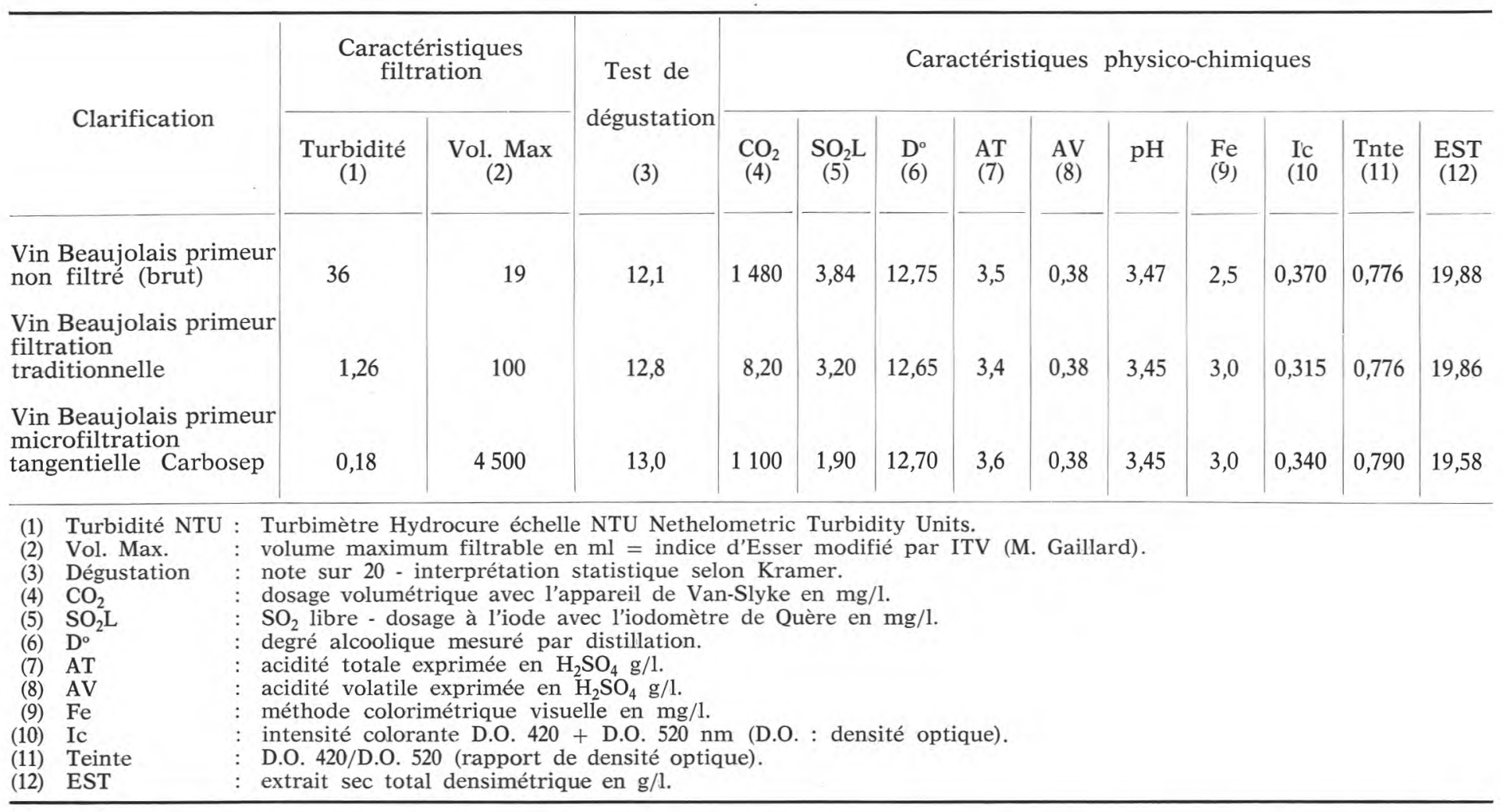


TABLEAU 5

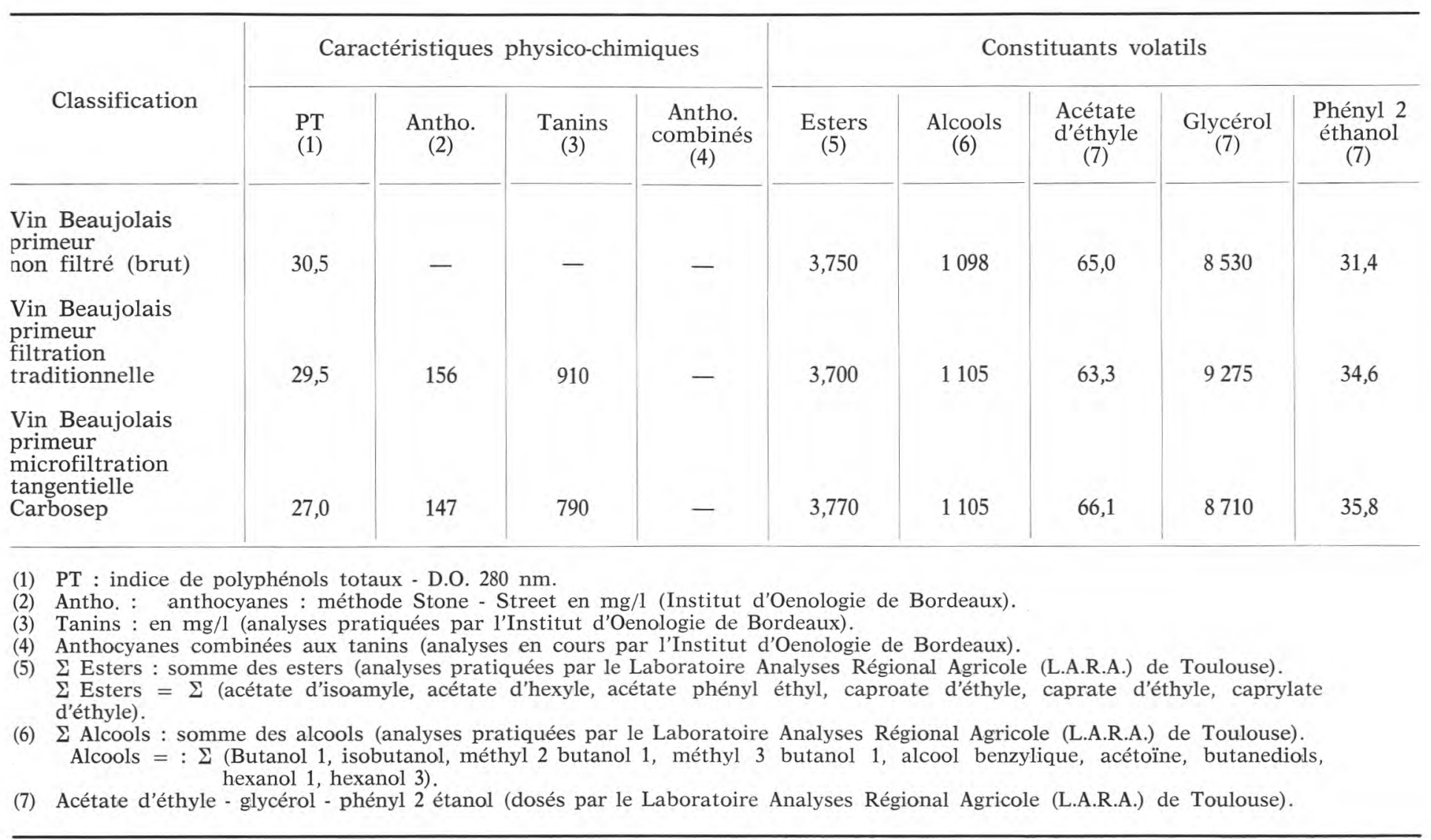


- Les tests de dégustation normalisés ne traduisent pas de différence significative.

- Les gaz dissous, les acidités volatiles et totales, les pH sont très sensiblement identiques.

- L'intensité colorante et la teinte sont légèrement plus élevées par le procédé de microfiltration tangentielle.

- Les anthocyanes et les tanins ont très légèrement diminué. Il est probable que les anthocyanes combinées aux tanins responsables de troubles ont été retenues par la membrane (analyses en cours).

- Les esters sont identiques, il faut rappeler qu'ils sont favorables à la dégustation en particulier l'acétate d'éthyle.

- Les alcools n'ont pas évolué par le procédé de microfiltration tangentielle; il faut rappeler qu'ils sont pour certains défavorables à la dégustation; le glycérol, agent mouillant des papilles gustatives n'est pas significativement modifié.

En conclusion les résultats présentés au regard de la qualité des vins obtenus par le procédé de microfiltration tangentielle Carbosep avec une nouvelle membrane adaptée à la clarification des boissons fermentées permet d'aborder le développement industriel de cette ligne de produit.

\section{CONCLUSION GENERALE}

Le procédé d'ultrafiltration Carbosep qui a apporté en 1980 un nouvel acquis technologique vis-à-vis des procédés mettant en jeu des fluides visqueux et des hautes températures a pu, grâce à la maîtrise de sa technologie membrane et des procédés, s'adapter à des procédés agro-alimentaires sur des fluides non visqueux à température modérée. Aujourd'hui prend naissance le procédé de microfiltration tangentielle Carbosep applicable en premier lieu à la filtration des boissons alcoolisées fermentées dont plus particulièrement le vin.

\section{Remerciements}

L'auteur remercie MM. Berge et Gaillard de l'Institut Technique du Vin. 